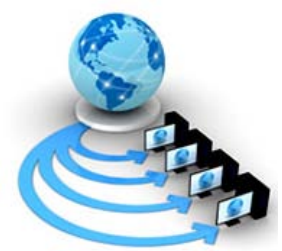

Volume 9, No. 2, March-April 2018

International Journal of Advanced Research in Computer Science

RESEARCH PAPER

\author{
Available Online at www.ijarcs.info
}

\title{
IMPROVING EFFICIENCY AND EFFECTIVENESS OF HIERARCHICAL CLUSTERING
}

\author{
P. Praveen \\ Assistant Professor \\ in Computer Science,S R Engineering College \\ and Research Scholar in Kakatiya \\ University,Warangal,Telangana,India
}

\author{
B. Rama \\ Assistant Professor in Department \\ of Computer Science, \\ Kakatiya University, Warangal, \\ Telangana, India.
}

\begin{abstract}
Clustering techniques will formulate the edifice of the groups by divide the instances in whichever a bottom-up or top-down fashion. These methods are divided into Divisive hierarchical clustering and Agglomerative hierarchical clustering. The nested combining of objects and corollary levels at which groupings change will be represented by the corollary of these methods. The clustered items are achieved by wounding dendrogram at the desired likeness rank. Here the Single linkage method is inter dependent on correlation of two clusters that are nearest points in different clusters. Complete linkage method is reliant on the correlation of two clusters that are least similar points in the different clusters. Average linkage method is reliant on the average of pair wise closeness between the points in two clusters. For choosing which strategies are most appropriate for a given dataset, here we proposed a ensemble based system
\end{abstract}

Keywords: Data Mining, Classification, Clustering Algorithms, Heart Attacks

\section{INTRODUCTION}

Some basic definitions are gathered from the clustering writing and given underneath

1."A Cluster is an arrangement of substances which are similar, and elements from various clusters are not alike."

2."A cluster is an accumulation of focuses in the space with the end goal that the separation between two focuses in the cluster is not asmuch as the separation between any point in the cluster and any point not in it."

3."Clusters might be portrayed as associated areas of a multidimensional space containing a moderately high thickness of focuses, isolated from other such districts by a locale containing a generally low thickness of focuses." And, after it's all said and done the cluster is an application subordinate idea, all clusters will be contrasted with deference with specific properties: thickness, fluctuation, measurement, shape, and partition. The cluster ought to be a tight and smaller high-thickness district of data indicates when thought about alternate territories of room. From minimization and snugness, it takes after that the level of scattering (difference) of the cluster is little. The state of the cluster isn't known from the earlier. It will be controlled by the utilized calculation and clustering criteria and partition characterizes the level of conceivable cluster cover and the separation to each other $[1,3,4]$.

Characterizing the attributes of a cluster, like giving a solitary, one of a kind and right definition, isn't a correct science (Copy right, 2006). Albeit distinctive creators underscore on various attributes, they do however concede to the principle measurements.

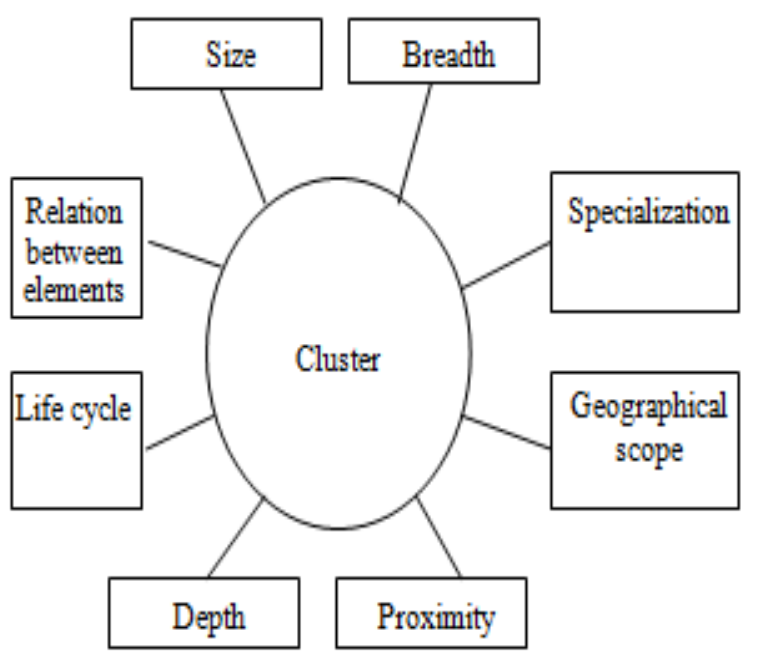

Figure 1 Main characteristics of a cluster

Limits of a cluster are not correct. Clusters shift in size, profundity and broadness. A few clusters comprise of little and some of medium and some of extensive in estimate. The profundity alludes to the range related by vertically connections. Besides, a cluster is portrayed by its broadness too. The breath is characterized by the range related by evenly connections $[2,5,8]$.

\section{LITERATURE REVIEW}

In 2009 Lan, Renxia Wan, Yuming Qin, Xiaoke Su proposed "A Fast Incremental Clustering Algorithm". In this paper, we propose a quick incremental clustering calculation by changing the sweep limit esteem progressively. This calculation will limit the quantity of definite clusters and peruses the first dataset just once. In the meantime the uniqueness measure considering the recurrence data of the characteristic esteems is presented. It can be utilized for the unmitigated data[6,11]. 
In 2010 Ranjit Biswas, Parul Agarwal, M. Afshar Alam proposed the profundity clarification of usage received for k-pragna, an agglomerative various leveled clustering method for straight out qualities [7,9].

In 2011 Hussain Abu-Dalbouh1 and Norita Md Norwawi proposed Bi-directional agglomerative various leveled clustering to make a pecking order base up, by iteratively combining the nearest match of data-things into one cluster. The outcome is an established AVL tree. The $\mathrm{n}$ leafs relate to enter data-things (singleton clusters) needs to $n / 2$ or $n / 2+1$ stages to converge into one cluster, compare to groupings of things in coarser granularities moving towards the root. The principle favorable position of proposed bi-directional agglomerative progressive clustering calculation utilizing AVL tree when contrasted and the other comparable agglomerative calculation is that, it has generally low computational necessities. The whole multifaceted nature of the proposed calculation is $\mathrm{O}(\log n)$ and required $(\mathrm{n} / 2$ or $\mathrm{n} / 2+1$ ) to cluster all data focuses in one cluster though the past calculation is $\mathrm{O}\left(\mathrm{n}^{2}\right)$ and need (n-1) ventures to cluster all data focuses into one cluster[10,13].

In 2012, Shengrui Wang, Dan Wei, Qingshan Jiang, Yanjie Wei proposed a strategy is which assesses clustering practically related quality arrangements and by phylogenetic investigation[14]. In this paper, an introduction of a novel approach for DNA succession clustering, in view of another arrangement likeness measure DMk which is separated from DNA groupings in light of the position and sythesis of oligonucleotide design. Diverse strategies for combinatorial issues frequently display exceptional execution that relies upon the solid issue example to be explained. The calculation will be expected to blend the qualities of numerous algorithmic methodologies via preparing a classifier that chooses or timetables solvers subject to the given occasion. Proposed calculation contrived a costdelicate various leveled clustering approach for building calculation portfolios. The observational examination demonstrated that including highlight mixes can enhance exhibitions daintily, at the cost of expanded preparing time, while combining cluster parts in light of cross-approval brings down prediction precision[4,15.]

\section{CLUSTERING METHODS}

Gigantic clustering techniques were created, each of which utilizes distinctive acceptance Standard. Raftery and Farley has proposed the isolating of clustering techniques into two gatherings - progressive and apportioning strategies. Kamber and Han arranging the techniques into extra three primary classes: thickness based strategies, demonstrate based clustering and matrix based strategies. In Estivill-Castro, 2000, another enlistment standard for various clustering strategies is introduced. We talk about some of them here[6,7].

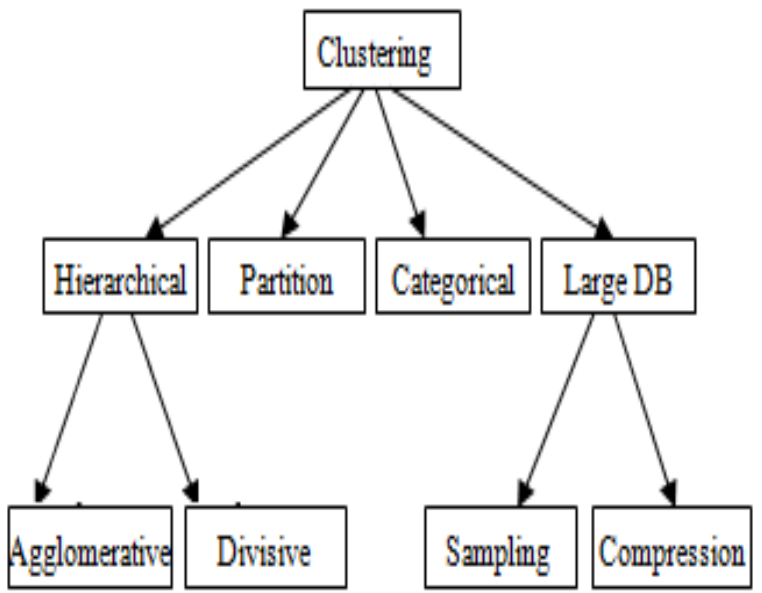

Figure 2: Clustering methods

In the wake of having picked the separation or likeness measure, we have to choose which clustering calculation to apply. There exists distinctive agglomerative systems and will be recognized by the way they characterize the separation from a recently framed cluster to a specific question, or to different clusters in the arrangement. The most prominent agglomerative clustering strategies incorporate the accompanying:

1) Single linkage (closest neighbor) - The separation between two clusters relates to the most brief separation between any two individuals in the two clusters.

2) Complete linkage - An oppositional way to deal with single linkage accept that the separation between two clusters depends on the longest separation between any two individuals in the two clusters.

3) Centroid - In this approach, the geometric focus (centroid) of each cluster is figured first. The separation between the two clusters meets the separation between the two centroids.

Here linkage calculation will deliver very surprising outcomes when utilized on the same dataset, as its particular properties. So it is exceptionally hard to choose which technique is to best to select data set. The clustering techniques for the most part create more valuable progressions and more conservative clusters than the singleconnect clustering strategies, yet the single-interface techniques are more versatile[9,10,11].

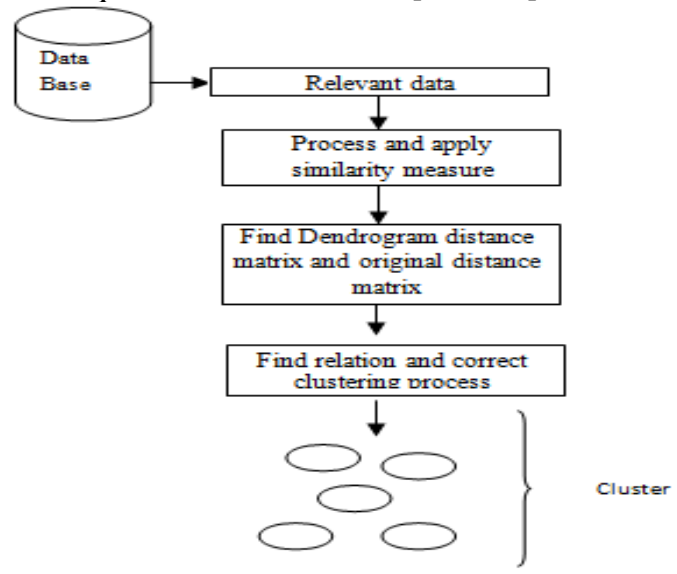

Figure 3: Architecture of proposed method 


\section{PROPOSED METHOD AND EXPERIMENTAL ANALYSIS}

1) Allocate every protest as respective group like c1, c2, c3,...cn everywhere $n$ is the number of items

2) Discover the separation framework D, utilizing any likeness measure

3) Find out the adjoining combine of cluster in the present clustering, say match (r), (s), as per $\mathrm{d}(\mathrm{r}, \mathrm{s})=$ mind (I, j) $\{\mathrm{I}$, is a question in cluster $\mathrm{r}$ and $\mathrm{j}$ in cluster $\mathrm{s}\}$

4) Combine the clusters (r) and (s) into a solitary gather to shape a blended cluster. Store consolidated articles with its comparing separation in Dendrogram remove Matrix.

5) Make the updation of separation framework D, by erasing the lines and segments comparing to clusters (r) and (s). Including another line and segment relating to the consolidated cluster(r, s) and old cluster (k) is characterized in this way: $d[(\mathrm{k}),(\mathrm{r}, \mathrm{s})]=\min \mathrm{d}[(\mathrm{k}),(\mathrm{r})], \mathrm{d}[(\mathrm{k}),(\mathrm{s})]$.For different lines and sections duplicate the comparing data from existing separation grid.

6) If all items are in one cluster, stop. Something else, go to stage 3 .

7) Find social incentive with single, finish and normal linkage strategies.

8) Generate right clusters.

We assess the execution of proposed calculation and contrast it and single linkage, finish linkage and normal linkage techniques. The trials are performed on Intel i64200U processor 4GB principle memory and RAM: 8GB OS:Windows 8.The calculations are executed in utilizing C\# Dot Framework Net dialect adaptation 4.0.1. Engineered datasets are utilized to assess the execution of the calculations. For looking at the execution of the proposed calculations, we actualize the single linkage and finish linkage technique. Our first examination depends on execution time and number of articles.

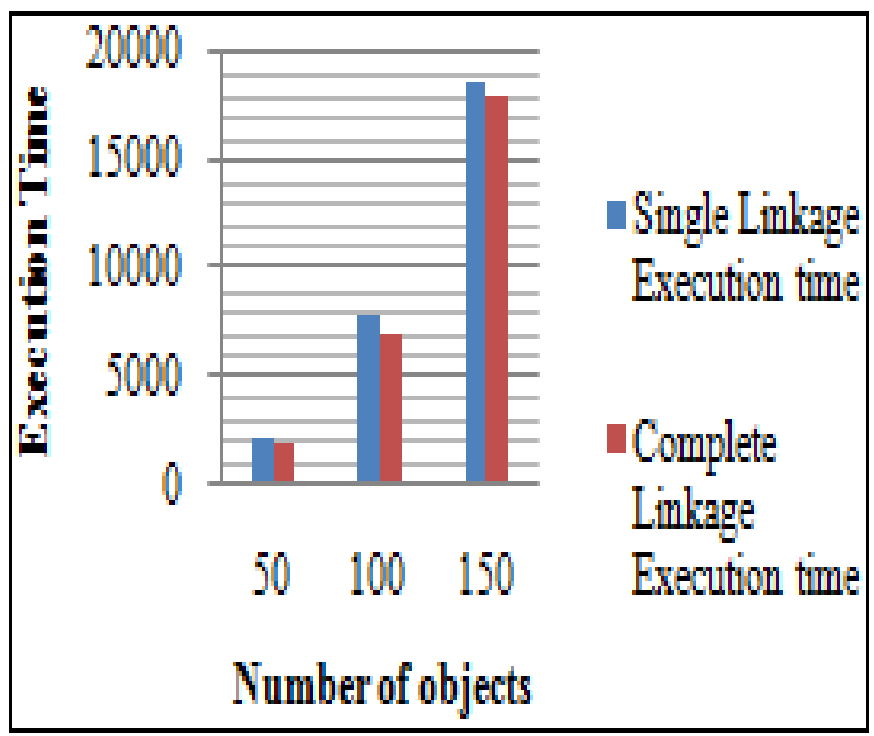

Figure4: Comparison graph with Execution time and number of objects

\section{CONCLUSION}

There are different classification techniques that can be used for the prevention and identification of heart disease. The concert of taxonomy techniques depends in the lead the type of dataset that have taken for performing trial. Classification techniques provide benefit to all the people such as healthcare insurers, patients, doctor and organizations who are engaged in healthcare industry. All these methods are compared with the basis of compassion, Specificity, precision, factual affirmative Rate, artificial affirmative Rate and fault Rate. The aim of each procedure is for predicting more precision in the incidence of heart ailment with least number of attributes.

\section{REFRENCES}

[1] Suneetha, K.Hari, Raj, "Modification of Gini Index Classification: A Case Study Of Heart Disease Dataset" International Journal on Computer Science and Engineering Vol. 02, No. 06, 2010, 1959-1965

[2] J O.P.Vyas and Sunita, Predictive Analysis in Health Data Mining Classifier "International Journal of Applications (0975 - 8887) Volume 4 - No.5, July 2010

[3] Khan, S.S., Ahmed, A., Cluster center initialization algorithm for k-means clustering, Pattern Recognition Letter, 25 (11) , pp. 1293-1302, 2004.

[4] P. Praveen, B. Rama and T. Sampath Kumar, "An efficient clustering algorithm of minimum Spanning Tree," 2017 Third International Conference on Advances in Electrical, Electronics, Information, Communicationand BioInformatics(AEEICB),Chennai,2017,pp.131-135.Doi 10.1109/AEEICB.2017.7972398R. Nicole, "Title of paper with only first word capitalized,” J. Name Stand. Abbrev., in press.

[5] Mai Shouman, Tim Turner, Rob Stocker "Using Decision Tree for Diagnosing Heart Disease Patients” Proceedings of the 9-th Australasian Data Mining Conference (AusDM'11), Ballarat, Australia

[6] Sunita Soni and O.P.Vyas "Fuzzy Weighted Associative Classifier: A Predictive Technique For Health Care Data Mining” International Journal of Computer Science, Engineering and Information Technology (IJCSEIT), Vol.2, No.1, February 2012

[7] Chaitrali S. Dangare and Sulabha S. Apte, PhD. Enhancement in Study of Heart Prediction System using Data Mining Classification Techniques, International Journal of Computer Applications (0975 - 888) Volume 47- No.10, June 2012

[8] Praveen P., Rama B. (2018) A Novel Approach to Improve the Performance of Divisive Clustering- BST. In:Satapathy S., Bhateja V., Raju K., Janakiramaiah B. (eds) Data Engineering and Intelligent Computing. Advances in Intelligent Systems and Computing, vol 542. Springer, Singapore.

[9] N S Nithyaand K Duraiswamy Gain ratio based fuzzy weighted association rule mining classifier for medical diagnostic interface Vol. 39, Part 1, February 2014, pp. 39-52. Indian Academy of Sciences

[10] P. Praveen and B. Rama, "An empirical comparison of Clustering using hierarchical methods and means," 2016 2nd International Conference on Advances in Electrical, Electronics, Information, Communication and BioInformatics (AEEICB), Chennai, 2016, pp. 445-449. Doi 10.1109/AEEICB.2016.7538328

[11] M. Sheshikala, D. Rajeswara Rao and R. Vijaya Prakash, Parallel Approach for Finding Co-location Pattern - A Map Reduce Framework, Twelfth International Multi-Conference on Information Processing-2016 (IMCIP-2016) 
M. Sheshikala, D. Rajeswara Rao and R. Vijaya Prakash, Computation Analysis for Finding Co-Location Patterns using Map-Reduce Framework, Indian Journal of Science and Technology, Vol 10(8), DOI: 10.17485/ijst/2017/v10i8/106709, February 2017.Jain, A. K., Data clustering: 50 years beyond k-means, Pattern Recognition Letters, vol. 31, no. 8, pp. 651-666, 2010.

[12] Murat E., Nazif C., Sadullah S., A new algorithm for initial cluster centers in k-means algorithm , Pattern Recognition Letters, 32,pp. 1701-1705, 2011.
[13] M Sheshikala, D Rajeswara Rao, R Vijaya Prakash, "A MapReduce Framework for Finding Clusters of Colocation Patterns-A Summary of Results" ,Advance Computing Conference (IACC), 2017 IEEE 7th International, Pages 12913

[14] R. Ravi Kumar, M. Babu Reddy and P. Praveen, "A review of feature subset selection on unsupervised learning," 2017 Third International Conference on Advances in Electrical, Electronics, Information, Communication and Bio-Informatics (AEEICB),

Chennai,2017,pp.163-167.doi: 